\title{
Engineering Education at the University of Toronto
}

Engineering education research at the University of Toronto is very active and growing. We currently have multiple faculty involved in individual and collaborative projects, and two Ph.D. candidates. Project topics areas include: teaching and assessment of teamwork and leadership; development of professional identity through portfolios; inverted classroom teaching methods; teaching effectively to diverse student populations; retention and grittiness; use of technology in the classroom and innovative pedagogy; outcomes based assessment methods; and collaborating on a study in critical thinking.

We maintain an informal research group called PEER (Practitioners in Engineering Education Research) that meets approximately monthly. PEER group is a community of practice intended to help members develop research projects and proposals, provide additional perspectives on results, and discussion of shared interests. This network has been very effective in leveraging individual expertise and supporting a vibrant research community.

Our Faculty is now taking the next step to establish a pathway for students pursuing a Ph.D. in engineering education. A task force to create a research based graduate program in engineering education was initiated in January and is currently developing a plan. In this session we will talk about the projects that are on-going and the plans for the future. 